\title{
A Birth and Death Process Model with Blocking Growth and its Numerical Simulation Research
}

\author{
Peng Yang ${ }^{1}$, Hua Liu ${ }^{1, *}$, Yumei Wei ${ }^{2}$, Ming $\mathrm{Ma}^{1}$ and Jianhua $\mathrm{Ye}^{1}$ \\ ${ }^{1}$ School of Mathematics and Computer Science, Northwest Minzu University, Lanzhou, 730030, Gansu, China \\ ${ }^{2}$ Experimental Center, Northwest Minzu University, Lanzhou, 730030, Gansu, China \\ ${ }^{*}$ Corresponding author
}

\begin{abstract}
In this paper, we established a model of the birth and death process with blocking growth. Based on the theory of stochastic process and the classical model of birth and death process, we calculated the expectation and variance of population. We also showed the influence of blocked items on the population by computer simulation. The application of the process in ecology has a certain practical value.
\end{abstract}

Keywords-stochastic process; process of birth and death; mathematical model; expectation; variance

\section{INTRODUCTION}

Stochastic processes can be grouped into two, namely discrete time stochastic processes and continuous time stochastic processes. One particular form of stochastic process with discrete space and continuous time is Poisson process that counts the number of events that occur at specified intervals. The development of the Poisson process is the process of birth, which is the number of counts that occurred at a specific time interval whose value depends on the number of events in the previous time interval. The birth process that has been widely discussed, namely pure birth process that can be seen in [1]. A birth and death process is a stochastic process in which jumps from a particular state, which are only allowed to the neighboring states. This property considerably simplifies the mathematical analysis, but the process remains applicable to numerous real-world systems. This was demonstrated by Miller and Cox [2] in 1987. In 2006, Novozhilov et al. [3] discussed the application of the birth and death process theory in ecology. In other studies, the quasi-stationary distribution of the birth and death processes of Doorn and Schoutens[4-5] and Pollet [6] were discussed in detail in 2003. Liu and Zhang [7] studied and discussed the quasi-stationary distribution of linear birth and death processes in two different ways in 2011. Some scholars discuss the recurrent and progressive nature of the birth and death process in random environments [8-10]. The birth and death process is a kind of random process that can be transferred to each other in a specific state. It only allows each state to transfer between neighboring states, describe the population to reproduce and die according to certain rules, and birth and death between each individual. It is independent of each other. This principle has brought great simplicity to mathematics calculations, but it has also been widely used in systems that consider the real world. The very beginning of population growth modeling, it had been clear that a most refined analysis must take into account the role of stochastic factors in the evolution of population. Mathematical field inspired by biology is the theory of birth and death process.

\section{Blocking BirTh AND DEATH PROcess ModeL}

If the death rate is not considered, the birth and death process degenerates into a purely biological process. At this time, the number of individuals in the population will grow indefinitely, ignoring the limitation of the environmental capacity, that is, the resource. The following assumptions are made: The birth rate of the population decreases as the population increases. The birth rate $\lambda_{n}=\beta\left(1-\frac{n}{K}\right)$, where $\beta$ is a constant related to the birth rate, and the environmental capacity $K$ is a constant. It can be seen from the structural birth rate $\beta\left(1-\frac{n}{K}\right)$, when the population number $n$ is closer to the environmental capacity $K$, the birth rate $\beta\left(1-\frac{n}{K}\right)$ is getting smaller and smaller, and when the environmental carrying capacity is reached, the birth rate is 0 , that is, it no longer grows. The definition of the birth and death process of retarded growth is as follows:

Let the state space of the homogeneous Markov process $\{X(t), t \geq 0\}$ to $I=\{0,1,2, \cdots\}$ and the transition probability to $p_{i j}(t)$, if

$$
\begin{gathered}
p_{n, n+1}(\Delta t)=\beta\left(1-\frac{n}{K}\right) \Delta t+o(\Delta t), \beta>0, \\
p_{n, n-1}(\Delta t)=\mu \Delta t+o(\Delta t), \mu>0 \\
p_{n, n}(\Delta t)=1-\left[\beta\left(1-\frac{n}{K}\right)+\mu\right] \Delta t+o(\Delta t), \\
p_{n, m}(\Delta t)=o(\Delta t),|n-m| \geq 2 .
\end{gathered}
$$

We call $\{X(t), t \geq 0\}$ the birth and death process of the growth of the block, where $\beta$ is the constant related to the birth rate, $\mu$ is the death rate, $\Delta t$ is the time interval, $n$ is the number of individuals in the current state, and $K$ is the environmental carrying capacity.

In order to study its probability $p_{n}(t)$, we can first find the equation, considering the case where the quantity is $n$. It is possible that there are $n-1$ individuals at time $t$ and then one during the $\Delta t$ interval; there are $\mathrm{n}+1$ individuals at time $t$ and 
then decrease by one during the $n+1$ interval; or at time $t$ there are already $n$ individuals, and then the individual who increases during the $t+\Delta t$ interval is the same as the declining individual, so that at the moment there are still $n$ individuals who have the following results:

$$
\begin{gathered}
p_{n-1, n}(\Delta t)=P\{N(t+\Delta t)-N(t)=1 \mid N(t)=n-1\} \\
=\beta\left(1-\frac{n-1}{K}\right) \Delta t+o(h) \\
p_{n+1, n}(\Delta t)=P\{N(t+\Delta t)-N(t)=-1 \mid N(t)=n+1\} \\
=\mu(n+1) \Delta t+o(\Delta t), \\
p_{n, n}(\Delta t)=P\{N(t+\Delta t)-N(t)=0 \mid N(t)=n\}, \\
=1-\left[\beta\left(1-\frac{n}{K}\right)+\mu\right] \Delta t+o(\Delta t) . \\
p_{n \pm j, n}(\Delta t)=o(\Delta t),|j| \geq 2 .
\end{gathered}
$$

and

$$
\begin{gathered}
p_{n}(t+\Delta t)=p_{n-1}(t) p_{n-1, n}(\Delta t) \\
+p_{n+1}(t) p_{n+1, n}(\Delta t)+p_{n}(t) p_{n, n}(\Delta t)+o(h) .
\end{gathered}
$$

We thus have that

$$
\begin{gathered}
p_{n}(t+\Delta t)=(n-1) \beta\left(1-\frac{n-1}{K}\right) \Delta t p_{n-1}(t) \\
+(n+1) \mu \Delta t p_{n+1}(t)+\left[1-n\left(\beta\left(1-\frac{n}{K}\right)+\mu\right) \Delta t\right] p_{n}(t)+o(\Delta t),
\end{gathered}
$$

Let $M(N \mid t)$ be the expected size of the population at time t,

$$
M(N \mid t)=\sum_{j=1}^{\infty} j p_{j}(t),
$$

and define the initial value

$$
M(N \mid 0)=i,
$$

that is, the expected value at the initial time is $i$, and

$$
\frac{d M(N \mid t)}{d t}=\sum_{j=1}^{\infty} j \frac{d p_{j}(t)}{d t} .
$$

For convenience, let $P_{j}$ instead of $P_{j}(t)$ into (1), get

$$
\begin{gathered}
\frac{d M(N \mid t)}{d t}=\sum_{j=1}^{\infty} j\left[(j-1) \beta\left(1-\frac{j}{K}\right) p_{j-1}(t)\right. \\
\left.+\mu(j+1) p_{j+1}(t)+j\left[1-\left(\beta\left(1-\frac{j-1}{K}\right)+\mu\right)\right] p_{j}(t)\right] \\
=\sum_{j=1}^{\infty}\left(j^{2} \beta P_{j-1}-\frac{j^{3} \beta-\beta j^{2}}{K} P_{j-1}-j \beta P_{j-1}\right. \\
\left.+j^{2} \mu P_{j+1}+j \mu P_{j+1}+\mu j^{2} P_{j}+j^{2} P_{j}\right) .
\end{gathered}
$$

Introduce a new variable, $m=j-1$, to rewrite the above equation as

$$
\frac{d M(N \mid t)}{d t}=\beta \sum_{m=0}^{\infty}\left(\frac{1}{K} m^{2}+m\right) P_{m}
$$

$$
+\mu \sum_{m=0}^{\infty}\left(2 m^{2}-m\right) P_{m}+\sum_{m=0}^{\infty} m^{2} P_{m}
$$

Where $\sum_{m=0}^{\infty} m^{2} P_{m}$ is Second-order origin moment. It can be obtained by integrating the equation into the integral operation,

$$
u(t)=\left(1+\frac{\beta}{K}+2 \mu\right) \cdot \frac{i(\beta+\mu)}{\mu-\beta}\left[t-\frac{1}{\mu-\beta} \cdot e^{(\beta-\mu) t}\right]
$$

Thus

$$
M=u(t) e^{(\beta-\mu) t}=\left(1+\frac{\beta}{K}+2 \mu\right) \cdot \frac{i(\beta+\mu)}{\mu-\beta}\left[t \cdot e^{(\beta-\mu) t}-\frac{1}{\mu-\beta}\right]
$$

For the calculation of the variance $\operatorname{var}(N \mid t)$, from the second-order origin moment $M_{2}(N \mid t)=\sum_{j=1}^{\infty} j^{2} P_{j}(t)$.

The equation is a first-order linear non-homogeneous equation and the corresponding homogeneous equation is solved. The result is

$$
M_{2}=C_{1} e^{\left[2(\beta-\mu)-\frac{\beta}{K}\right] t}
$$

Let $M_{2}=u(t) e^{\left[2(\beta-\mu)-\frac{\beta}{K}\right] t}$, It can be obtained by integrating the equation into the integral operation:

$$
\begin{gathered}
\int d u=\int\left(i \beta \cdot \mathrm{e}^{\left[2(\beta-\mu)-\frac{\beta}{K}\right] t}-\frac{\beta \cdot i(\beta+\mu) t}{K(\mu-\beta)} \cdot \mathrm{e}^{2(\beta-\mu)-\frac{\beta}{K}}\right. \\
\left.-\frac{2 \beta}{K} \cdot\left[\frac{i(\beta+\mu)}{\mu-\beta} \cdot \mathrm{e}^{\left[2(\beta-\mu)-\frac{\beta}{K}\right] t}-\frac{1}{2}\left(i+3 \frac{i(\beta+\mu)}{\mu-\beta}\right) \cdot \mathrm{e}^{\left[2(\beta-\mu)-\frac{\beta}{K}\right] t}\right]\right) d t
\end{gathered}
$$

Thus

$$
\begin{gathered}
M_{2}=\frac{i}{\frac{1}{K}-2}-\frac{i \beta(\beta+\mu)}{K(\beta-\mu)}\left[\frac{1}{2(\beta-\mu)-\frac{\beta}{K}}\right. \\
\left.\cdot e^{2(\beta-\mu)-\frac{\beta}{K}}-\frac{1}{2(\beta-\mu)-\frac{\beta}{K}} \cdot e^{2(\beta-\mu)-\frac{\beta}{K}}\right] \\
-\frac{2 \beta}{K} \cdot\left[\frac{i(\beta+\mu)}{\mu-\beta} \cdot \frac{1}{2(\beta-\mu)-\frac{\beta}{K}} .\right. \\
\left.+\frac{\beta}{K}\left(i+3 \frac{i(\beta+\mu)}{\mu-\beta}\right) \cdot \frac{1}{2(\beta-\mu)-\frac{\beta}{K}}\right]+C_{2}
\end{gathered} .
$$

Where $C_{2}$ is an integral constant, and this constant is calculated as the second-order moment $M_{2}$ at the time $t$ of the birth and extinction process with a block. According to the relationship between the variance and the second-order origin moment we got

$$
\operatorname{var}(N \mid t)=\frac{i}{\frac{1}{K}-2}-\frac{i \beta(\beta+\mu)}{K(\beta-\mu)}\left[\frac{1}{2(\beta-\mu)-\frac{\beta}{K}}\right.
$$




$$
\begin{gathered}
\left.\cdot t e^{\left[2(\beta-\mu)-\frac{\beta}{K}\right] t}-\frac{1}{2(\beta-\mu)-\frac{\beta}{K}} \cdot e^{\left[2(\beta-\mu)-\frac{\beta}{K}\right] t}\right] \\
-\frac{2 \beta}{K} \cdot\left[\frac{i(\beta+\mu)}{\mu-\beta} \cdot \frac{1}{2(\beta-\mu)-\frac{\beta}{K}}\right. \\
\left.+\frac{\beta}{K}\left(i+3 \frac{i(\beta+\mu)}{\mu-\beta}\right) \cdot \frac{1}{2(\beta-\mu)-\frac{\beta}{K}}\right]+C_{2}
\end{gathered} .
$$

That is, the desired expectation is:

$$
\begin{gathered}
E[N \mid t]=M(N \mid t)=\left(1+\frac{\beta}{K}+2 \mu\right) \\
\cdot \frac{i(\beta+\mu)}{\mu-\beta}\left[t \cdot e^{(\beta-\mu) t}-\frac{1}{\mu-\beta}\right]+i+\left(1+\frac{\beta}{K}+2 \mu\right) \cdot \frac{i(\beta+\mu)}{(\mu-\beta)^{2}}
\end{gathered}
$$

According to the relationship between the variance and the second-order origin moment we got

$$
\begin{gathered}
\operatorname{var}(N \mid t)=\frac{i}{\frac{1}{K}-2}-\frac{i \beta(\beta+\mu)}{K(\beta-\mu)} \\
-\frac{2 \beta}{K} \cdot\left[\frac{i(\beta+\mu)}{\mu-\beta} \cdot \frac{1}{2(\beta-\mu)-\frac{\beta}{K}}\right. \\
\left.+\frac{\beta}{K}\left(i+3 \frac{i(\beta+\mu)}{\mu-\beta}\right) \cdot \frac{1}{2(\beta-\mu)-\frac{\beta}{K}}\right]+C_{2} .
\end{gathered}
$$

From initial conditions

$$
\operatorname{var}(N \mid 0)=0 .
$$

Than

$$
\begin{gathered}
\operatorname{var}(N \mid t)=i-\frac{i \beta(\beta+\mu)}{K(\beta-\mu)}\left[\frac{1}{2(\beta-\mu)-\frac{\beta}{K}} \cdot t e^{\left[2(\beta-\mu)-\frac{\beta}{K}\right] t}\right. \\
\left.-\frac{1}{2(\beta-\mu)-\frac{\beta}{K}} \cdot e^{\left[2(\beta-\mu)-\frac{\beta}{K}\right] t}\right]-\frac{1}{\left[2(\beta-\mu)-\frac{\beta}{K}\right]^{2}}
\end{gathered}
$$

In summary, the expectation and variance of the block birth and death process are obtained. This result introduces a retardation term. Therefore, it is more realistic than the general birth and death process.

\section{COMPUTER SIMULATION}

Computer simulation has important reference meanings for the study of complex ecological mathematical models. The classical model of birth and death process does not contain blocking items, that is, environmental resources are infinite, and individuals within the population do not suffer from resource issues. Growth is limited. Since the population change in the model of birth and death process is random, a simulation result cannot explain that the result is consistent with reality. At the same time, the block birth and death process model in this paper contains retarded items, that is, the growth rate is related to the environmental carrying capacity. The function, so the simulation using classical birth and death process and block birth and death process comparative study, multiple simulation results.

\section{CONCLUSION}

First, set the initial population of each parameter value to $N=10$, the parameter $\lambda_{0}=\beta=0.3$ related to the birth rate of the population, the environmental carrying capacity $K=50$, and the ending time of the loop to 1000 . The average number and duration of the population are rounded and rounded. According to computer simulations, the following two tables and images can be drawn. Each case is simulated several times. The result of ten consecutive random simulations is listed in the table.

When the constant of the population itself and the birth rate are greater than the mortality $(\lambda>\mu)$, multiple simulations show that the average number of populations of the unblocked model exceeds the environmental capacity five times, since the initial number is 10, At the same time, the number of simulations is limited, and the simulation results show that the final number will exceed the unlimited growth of environmental capacity. In the retarded model, the population tends to stabilize with time. When the environmental carrying capacity is reached, the population is stable.Fig I is a curve that reflects the change in the number of populations. The model curve with no block has always been in a rising state, ie, the number of populations has grown without limit. In the blocked model, the curve maintains a relatively stable state after it grows to a certain extent, and does not continue to grow.

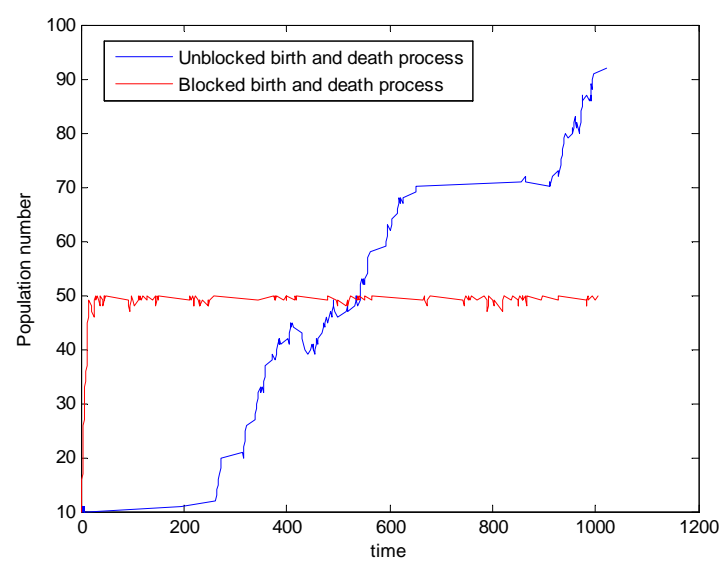

FIGURE I. POPULATION SIZE SIMULATION OF BIRTH AND DEATH PROCESSES WITHOUT BLOCKING 


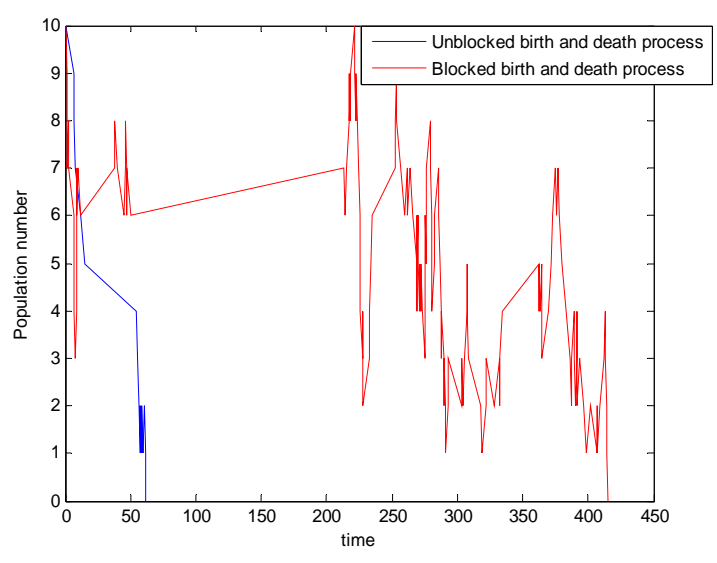

FIGURE II. POPULATION SIZE SIMULATION OF BIRTH AND DEATH PROCESSES WITHOUT BLOCKING

We can see that $(\lambda<\mu)$ when the population's own constant associated with the birth rate is less than the mortality rate, the population is constantly changing and eventually extinct. But in the unblocked model, the population has a longer duration. Fig II can be seen more directly in the trend of changes in the number of populations in the two models, and finally the model with blockage extinct.

It is easy to see from the results that in a resourceconstrained ecosystem, the possibility of species extinction will increase as resources become scarce and mortality increases. In order to protect endangered species, in addition to controlling their birth rate and mortality, they must also satisfy the resources needed for their species' survival. For example, food, living space, etc.

In this paper, only the blockade item is added to the birth item to limit the unlimited growth of the population when the model is established, and the mortality rate is still considered as a constant. In the later model, the mortality rate can be improved as a specific function that better reflects the changes in reality. Or when considering the birth item, the number of female individuals can be considered separately because the increase in the population size is more relevant to female individuals.

In this study, we have shown that acccording to our model for competitive dynamics of a blocking growth population, the extinction of the native species is minimized for number. One might also be interested in long-term measure of time during which the native species is absent or the average population size other native species. The construction of stochastic model of birth and death process with blocking can be done using the properties of Poisson process and some mathematical manipulations.

\section{DISCUSSION}

For further research, we can expand the scope of our models, by adding the growth rate of the female population, because population growth is also affected by the number of female. We can also apply this formula to model the growth of a animal in an area.
With the help of our birth and death process with blocking model, we quantified the feedback between ecological and genetic effects of the introduced species on the native competitor. This feedback is synergistic in the sense that ecological and genetic effects enhance each other: A reduction in population size causes a reduction in genetic diversity and effects enhance each other: A reduction in population size causes a reduction in genetic diversity and this reduction in diversity can lead to further population decline in a changing environment. We found that this eco-genetic feedback is particularly strong for small intensities of competition between the introduced and the native species.

\section{ACKNOWLEDGMENT}

This work is supported by the Fundamental Research Funds for the Central Universities (31920180116, 31920180044), the National Natural Science Foundation of China (31260098, 11361049, 31560127), the Program for Yong Talent of State Ethnic Affairs Commission of China (No.[2014]121), the Gansu Provincial first-class discipline program of Northwest Minzu University, the Central Universities Fundamental Research Funds for the Graduate Students of Northwest Minzu University(Yxm2018117), the 2018 laboratory opening project of Northwest Minzu University (SYSKF-2018225, SYSKF2018236).

\section{REFERENCES}

[1] Karlin, Taylor, introduction to stochastic modeling [J]. 2010, 36(4):387388.

[2] Cox D R, Miller H D, Weiss G, et al. The Theory of Stochastic Processes [M]. Springer-Verlag, 1979.

[3] Novozhilov A S, Karev G P, Koonin E V. Biological applications of the theory of birth-and-death processes.[J]. Briefings in Bioinformatics, 2006, 7(1):70-85.

[4] Van Doorn. Quasi-Stationary Distributions and Convergence to QuasiStationarity of Birth-Death Processes [J]. Advances in Applied Probability, 1991, 23(4):683-700.

[5] Schoutens W. Birth and death processes, orthogonal polynomials and limiting conditional distributions [J]. Mathematical Scientist, 2000, 25(2):87-93.

[6] Damian Clancy, Philip K. Pollett. A Note on Quasi-Stationary Distributions of Birth-Death Processes and the Sis Logistic Epidemic [J]. Journal of Applied Probability, 2003, 40(3):821-825.

[7] Liu W, Zhang H. Quasi-Stationary Distributions in Linear Birth and Death Processes [J]. Journal of Mathematics Research, 2011, 3(1).345360

[8] Cohen J E. Mathematics Is Biology's Next Microscope, Only Better; Biology Is Mathematics' Next Physics, Only Better [J]. Plos Biology, 2004, 2(12):439.

[9] Cougburn Ritter. Random Walks in a Random Environment [J].Appl. Probab, 1981, (18):19-30.

[10] Jagers P. The Growth and Stabilization of Populations [J]. Statistical Science, 1991, 6(3):269-274.M. Young,The Technical Writer's Handbook. Mill Valley, CA: University Science, 1989. 